\title{
Improving assessment and management of large non- pedunculated colorectal lesions in a Western center over 10 years: lessons learned and clinical impact
}

\section{다 (1) $(-)$}

\author{
Authors \\ Giuseppe Vanella, Chiara Coluccio, Giulio Antonelli, Stefano Angeletti, Federica Micheli, Antonio Barbato, Gaia De \\ Rossi, Alessandro Marchetti, Elena Mereu, Paola Pepe, Vito Domenico Corleto, Giancarlo D’Ambra, Maurizio Ruggeri, \\ Emilio Di Giulio
}

\begin{abstract}
Institution
Endoscopy Unit, Sant'Andrea Hospital, Faculty of Medicine and Psychology, Sapienza University of Rome, Italy
\end{abstract}

submitted 19.2.2020

accepted after revision $\quad 28.5 .2020$

\section{Bibliography \\ Endoscopy International Open 2020; 08: E1252-E1263 \\ DOI 10.1055/a-1220-6261 \\ ISSN 2364-3722 \\ (c) 2020. The Author(s). \\ This is an open access article published by Thieme under the terms of the Creative Commons Attribution-NonDerivative-NonCommercial License, permitting copying and reproduction so long as the original work is given appropriate credit. Contents may not be used for commecial purposes, or adapted, remixed, transformed or built upon. (https://creativecommons.org/licenses/by-nc-nd/4.0/)}

\section{Corresponding author}

Giuseppe Vanella, MD, Digestive Endoscopy Unit -

Sant'Andrea University Hospital, Sapienza, University of Rome - Faculty of Medicine and Psychology, Via di Grottarossa, 1035-1039-00189 Rome, Italy

Fax: + 390226435609

g.e.vanella@gmail.com

Supplementary material is available under https://doi.org/10.1055/a-1220-6261

\section{ABSTRACT}

Background and study aims Outcomes of endoscopic assessment and management of large colorectal (CR) nonpedunculated lesions (LNPLs) are still under evaluation, especially in Western settings. We analyzed the clinical im- pact of changes in LNPL management over the last decade in a European center.

Patients and methods All consecutive LNPLs $\geq 20 \mathrm{~mm}$ endoscopically assessed (2008-2019) were retrospectively included. Lesion, patient, and resection characteristics were compared among clinically relevant subgroups. Multivariate logistic regression (for predictors of submucosal invasion [SMI] and recurrence), Kaplan-Meier curves and ROC curves (for temporal cut-offs in trends analyses) were used. Results A total of $395 \mathrm{LNPLs}$ were included $(30 \mathrm{~mm}$ [range 20-40]; $\mathrm{SMI}=9.6 \%$; primary endoscopic resection [ER]= $88.4 \%$ ). Pseudo-depression and JNET classification independently predicted SMI beyond single morphologies/location. After complete ER, involvement of ileocecal valve/ dentate line, piece-meal resection and high-grade dysplasia independently predicted recurrence. Rates of 5-year recurrence-free, surgery-free and cancer-free survival were $77.5 \%, 98.6 \%$ and $100 \%$, respectively, with $93.8 \%$ recurrences endoscopically managed and no death attributable to ER or CR cancer (versus 3.4\% primary surgery mortality). ROC curves identified the period $\geq 2015$ (following Endoscopic Submucosal Dissection [ESD] introduction and education on pre-resective lesion assessment) as associated with improved lesions' characterization, increased en-bloc resection of SMI lesions ( $87.5 \%$ vs $37.5 \%$; $p=0.0455)$, reduced primary surgery $(7.5 \%$ vs $16.7 \%$; $=0.0072)$, surgical referral of benign lesions ( $5.1 \%$ vs $14.8 \% ; p=0.0019$ ), and recurrences.

Conclusions ESD introduction and educational interventions allowed ER of more complex lesions, offset by increased complementary surgery for complications or intrinsic histological risk. Nevertheless, overall, they have reduced surgery demand and increased appropriateness and safety of LNPL management in our center.

\section{Introduction}

Large $(\geq 20 \mathrm{~mm})$ non-pedunculated colorectal (CR) lesions (LNPLs) harbor a significant risk of malignancy and are associated with technical difficulty in achieving complete endoscopic resection (ER) [1-3]. Technological improvements (e.g. endoscopic submucosal dissection [ESD]) and a deeper comprehension of the biological behavior of cancer precursors have enabled endoscopists to consider ER for lesions of increasing complexity. However, these procedures have a longer training 
curve, are more resource-intense, carry a higher risk of adverse events (AEs), and their long-term advantages over surgery are still debated [4-8].

Selection of the correct therapeutic approach strictly relies on the capability of endoscopists to identify lesions at higher risk of superficial submucosal invasion (SMI) that warrant enbloc (EB) resection to ensure precise histological evaluation and endoscopic curability, but currently available predictors have suboptimal performance [1, 7, 9-12].

Moreover, long-term results of endoscopic management are heterogeneous, mainly derived from Eastern experience, where ESD is more widely available and outcomes have proven to be different from those in Western series $[8,13-16]$.

Finally, analysis of quality improvement in this area is usually restricted to learning curves of single resection techniques [17, 18].

Our aim was to describe endoscopic assessment and management of all consecutive LNPLs treated in a single European referral center over 10 years, to analyze: 1) pre-resection prediction of SMI; 2) outcomes of treatment according to resection technique; and 3) temporal trends of clinically relevant performance measures while expertise improved.

\section{Patients and methods}

A retrospective search of prospectively collected endoscopic and histological databases of Sant'Andrea Hospital, an academic, referral center for endoscopic management of CR lesions, was executed from January 2008 to April 2019.

Procedures were executed by the same five endoscopists (SA, VDC, GDA, MR and EDG) over time, all of whom were experienced in endoscopic luminal resection. LNPLs were treated through: 1) endoscopic mucosal resection (EMR) when the lesion was lifted through submucosal fluid injection and finally resected through a snare; 2 ) ESD, when fluid submucosal injection was used to expand the submucosal space, which was then accessed through an endoscope equipped with a transparent hood for careful manual dissection through a knife; and 3) hybrid procedures, when a part of the lesion was dissected through a knife and another part resected through snaring (usually a peripheral dissection and a central snaring $[19,20]$ ).

All consecutive LNPLs $\geq 20 \mathrm{~mm}$ were included, notwithstanding primary treatment (e.g. surgical versus [vs.] endoscopic, EMR vs. ESD), provided that endoscopic assessment and definitive histology were both available.

Excluded lesions were: 1)<20 mm; 2) pedunculated; 3) had obvious deep invasion (e.g. ulcerated); or 4) were in patients with inflammatory bowel diseases or genetic syndromes.

Morphology and superficial pattern were extracted from the databases and all images and videos were reevaluated by agreement of four trained endoscopists unaware of histology. Each lesion was categorized using JNET classification [21,22] for vascular pattern and one modified Paris classification [23], to further include sessile lesions (sessile [S]; sessile with pseudodepression [S-PD]; LST granular, homogeneous [LST-GH] or mixed [LST-GM]; LST non-granular, flat-elevated [LST-NG-FE] or pseudodepressed [LST-NG-PD]). Pseudodepression [23] (PD) was furthermore independently dichotomised as present/ absent. As for lesion size, an endoscopic estimate initially was used as a reference, but was subsequently systematically compared to the histopathological report, with any significant disagreement leading to lesion exclusion.

The study was approved by local Institutional Review Board.

\section{Definitions}

Histological diagnosis followed World Health Organization definitions [24].

Submucosal invasion (SMI) was categorized as "high-risk" for lymph node metastasis when at least one of the universally adopted histological risk factors (i.e. G3 differentiation, submucosal invasion $>1000 \mu \mathrm{m}$, lymphovascular invasion and tumor budding) $[2,25]$ was identified, or "low-risk" when all the above-mentioned parameters were excluded. In case of SMI lesions, we defined "accurate histological stratification" when all risk factors $[2,25]$ were reported in the histological report.

Surgery was defined as "primary" when indicated soon after endoscopic assessment without any attempt of endoscopic resection, and "complementary" when executed after primary endoscopic resection.

ER was deemed "curative" after a macroscopically complete resection of lesions with intramucosal dysplasia or "low-risk" SMI and "non-curative" after an incomplete resection, a resection of "high-risk" SMI lesions or SMI lesions resected piecemeal (PM) [26].

Post-treatment follow-up (FU) data were registered: recurrence; surgery for recurrences; and cancer-specific mortality. FU was analyzed only for non-surgically resected patients, endoscopically treated before June 2018 (allowing > 12 months FU at analysis time). At the moment of data extraction, all patients were contacted through phone calls to update clinical follow-up and register subsequent endoscopic examinations performed in other centers. Endoscopic follow-up was required for analysis of recurrence. For surgically treated and lost-to-FU patients, data on mortality were retrieved from health databases.

We defined as "potentially preventable" surgeries executed for 1 ) primary referral of benign lesions (BLs); 2) PM resection of invasive lesions (ILs); 3) complications; and 4) recurrences.

\section{Statistical analysis}

Categorical variables were compared through the $\mathrm{X}$-squared test, while continuous variables with t-test or Mann-Whitney test, as appropriate.

When analyzing associations between continuous variables and an outcome of interest, receiver operating characteristic (ROC) curves were used to identify cut-offs discriminating different outcome prevalences, which were used to dichotomize continuous variables.

Multivariate logistic regression models were built using a stepwise method, with a $P$ - to enter $<0.05$ and a $P$ to stay $<0.1$. Results were expressed as odds ratio (OR) and $95 \%$ confidence interval $(\mathrm{Cl})$.

Recurrence-, surgery-, and cancer-free survival was evaluated using Kaplan-Meier curves. The influence of independent variables was evaluated by stepwise Cox-proportional-hazards 
regression. Outcomes were expressed as adjusted survival probabilities at mean of covariates.

For trends analysis, we paired each procedure with a time lag (years) from inception of the study and analyzed this continuous variable in ROC curves to explore cut-offs separating periods with different prevalences of binary performance measures (i. e. presence/absence of an outcome).

$P<0.05$ was considered statistically significant.

\section{Results}

A total of 395 patients (age 70 [interquartile range (IQR) 6276], male 57.5\%) with LNPLs (30 mm [IQR 20-40]) were evaluated during the study period ( $\triangleright$ Table 1 ).

Identification/exclusion of patients is summarized in Supplementary Table 1.

\section{Characteristics of included lesions}

\section{Differences according to location}

Colonic vs rectal lesions are compared in Supplementary Table 2, showing a significantly different distribution of morphological types and histologies through the colon.

Invasive lesions (ILs) versus benign lesions (BLs)

38/395 (9.6\%) lesions presented as submucosally invasive.

Characteristics of ILs and BLs are compared in > Table 1 (significant differences shown in Supplementary Fig.1). ILs were more frequent among older patients ( $\geq 74$ years, $P=0.03$ ) and among larger lesions ( $\geq 40 \mathrm{~mm}, P=0.001)$, distal lesions $(P=$ $0.0134)$ and those with pseudodepression $(P<0.0001)$. SMI was significantly different among the various morphologies, ranging from $1.9 \%, 2.7 \%$ and $7.6 \%$ of LST-GH, LST-NG-FE and sessile lesions, to $11.9 \%, 28.1 \%$ and $35 \%$ of LST-GM, S-PD and LST-NG-PD respectively $(P<0.0001)$.

JNET classification was available for 294 lesions (74.4\%). All lesions with Pattern 1 (21/21) corresponded to a serrated histology. All lesions with Pattern 3 (3/3) were invasive beyond muscolaris propria. $53.6 \%$ of JNET $2 b$ and $3.3 \%$ of JNET 2a lesions were found with SMI $(P<0.0001$, > Table 1 and Supplementary Fig. 1).

\section{Predictors of invasiveness}

We used variables associated to SMI at univariate analysis to build multivariate logistic regression models. In the simplest model (M1), considering pre-resection variables available for all lesions, pseudodepression $(\mathrm{OR}=7.5[\mathrm{Cl} 3.6-15.6])$ and dimension $\geq 40 \mathrm{~mm}(\mathrm{OR}=2.4[\mathrm{Cl} 1.2-5]$ ) were the only independent predictors of SMI, beyond other single morphological characteristics. The addition of JNET classification and non-lifting sign to this model $(M 2)$ resulted in only a JNET $2 b / 3(O R=51.8$ [Cl 12.5-215.5]) and PD (OR=4.6 [Cl 1.1-19.7]) independently predicting SMI ( $>$ Table 1 and Supplementary Fig. 1).

As diagnostic tests (Supplementary Table 3), both predictive models had a high negative predictive value for SMI (96.9\% for M1 and $98.2 \%$ for M2), with a lower positive predictive value.

\section{Management}

After endoscopic assessment, primary surgical referral was $11.6 \%$, more frequently for ILs than BLs (31.6\% vs $9.5 \%$; $P=$ 0.0002)

Among patients in whom an ER was first attempted $(\mathrm{N}=$ 349), there were 31 incomplete resections (8.9\%; 9 endoscopically retreated), and nine perforations (2.6\%; 6 endoscopically managed). Both events were significantly more frequent among ILs ( $>$ Table 2 )

Need for complementary surgery after ER was significantly higher among ILs than BLs (69.2\% vs $5.6 \%, P<0.0001)$, due to the relatively higher rate of adverse events, but also to presence among SMI lesions of two (7.6\%) piecemeal resections and seven (26.9\%) high-risk histologies after a complete ER.

Among ILs managed by primary endoscopic resection ( $\mathrm{N}=$ 26), eight patients did not undergo subsequent surgery (surgical sparing $=30.8 \%$ ), four for being unfit despite high-risk histology while four (15.4\%) had curative ERs.

\section{Resection technique}

Lesions that were larger, flat, with more dangerous morphology (e. g. nodules/PD) or superficial pattern (i. e. JNET 2B/3), involving "risky hotspots" (i. e. dentate line, ileocecal valve or appendix) and with more advanced histology were relatively more frequent among patients treated through ESD rather than EMR (Supplementary Table 4).

There was a higher rate of EB resection among ESDs (Supplementary Table 4). Incomplete resections, recurrences and surgeries for recurrence were similar among the two techniques. There was a trend towards a more frequent need for complementary surgery following ESD, due to the relatively higher rate of surgery for perforation ( $3.6 \%$ vs $0.3 \%, P=0.01$ ) and for high-risk histology after a complete endoscopic resection (7.5\% vs $1.7, P=0.01)$. Conversely, among ESDs there was a higher prevalence of ILs not undergoing complementary surgery $(7.5 \%$ vs $1.4 \%, p=0.007)$ and of ILs with a curative endoscopic resection ( $5.6 \%$ vs $0.3 \%, \mathrm{P}=0.0007$; Supplementary Table 4).

\section{Follow-up}

Endoscopically managed patients

Among non-operated patients with a complete ER $(\mathrm{N}=307)$, 244 underwent any clinical FU while 14 (5.4\%) were lost-to-FU.

\section{Recurrence}

Among patients with endoscopic FU $(\mathrm{N}=204$; median 16 months [IQR 10-34]), recurrence was found in 42 (20.5\%) after a median interval of 7 months [IQR 4-13] from index procedure. Considering also patients experiencing $>1$ recurrence ( $N=19$, maximum 3 per patient), endoscopic treatment of recurrence was possible in $60 / 64$ cases $(93.8 \%)$, while 4 patients $(9.5 \%)$ were finally sent to surgery.

A ROC curve identified a dimension $\geq 30 \mathrm{~mm}$ as best (area under the curve $[A U C]=0.702, P<0.0001$ ) separating recurrent $(28.9 \%)$ vs non-recurrent $(9.9 \%)$ lesions $(P=0.0008)$. Risk 
- Table 1 Characteristics of included lesions, comparisons based on histology (invasive vs. benign) and multivariate analysis of predictors of submucosal invasion.

\begin{tabular}{|c|c|c|c|c|c|}
\hline Variable & \multicolumn{5}{|l|}{$\begin{array}{l}\text { All patients } \\
(\mathrm{N}=395)\end{array}$} \\
\hline Age [IQR], years & \multicolumn{5}{|l|}{$70[62-76]$} \\
\hline Male sex & \multicolumn{5}{|l|}{$228(57.7 \%)$} \\
\hline Dimensions [IQR], mm & \multicolumn{5}{|l|}{$30[20-40]$} \\
\hline $\begin{array}{l}\text { Reason of the exam: } \\
\text { screening }\end{array}$ & \multicolumn{5}{|l|}{$96(24.3 \%)$} \\
\hline \multirow[t]{3}{*}{ Histology } & \multicolumn{5}{|l|}{ LGD: 221 (55.9\%) } \\
\hline & \multicolumn{5}{|l|}{ HGD: $136(34.4 \%)$} \\
\hline & \multicolumn{5}{|l|}{ T1 or superior: 38 (9.6\%) } \\
\hline Variable & $\begin{array}{l}\text { Invasive Lesions } \\
(\mathrm{N}=38)\end{array}$ & $\begin{array}{l}\text { Benign Lesions } \\
(\mathrm{N}=357)\end{array}$ & $P$ value & $\begin{array}{l}\text { Multivariate } \mathrm{M}^{1}{ }^{1} \\
\text { OR }[\mathrm{Cl}]\end{array}$ & $\begin{array}{l}\text { Multivariate } \mathrm{M}^{2} \\
\text { OR }[\mathrm{Cl}]\end{array}$ \\
\hline Age $\geq 74$ years $^{3}$ & $19(50.0 \%)$ & $114(31.9 \%)$ & $0.0252^{4}$ & NS & NS \\
\hline Sex (Male) & $22(57.9 \%)$ & $206(57.7 \%)$ & 0.9819 & & \\
\hline Colon: Rectum & $27(71.1 \%): 11(28.9 \%)$ & $268(75.1 \%): 89(24.9 \%)$ & 0.5887 & & \\
\hline Proximal: Distal & $16(42.1 \%): 22(57.9 \%)$ & $224(62.7 \%): 133(37.3 \%)$ & $0.0134^{4}$ & NS & NS \\
\hline Dimensions $\geq 40 \mathrm{~mm}^{3}$ & $19(50.0 \%)$ & $91(25.5 \%)$ & $0.0014^{4}$ & OR 2.4 [1.2-5] & NS \\
\hline Sessile: LST & $16(42.1 \%): 22(57.9 \%)$ & $108(30.4 \%): 247(69.9 \%)$ & 0.1413 & & \\
\hline \multicolumn{4}{|l|}{ Composite Morphology } & NS & NS \\
\hline.$S$ & $7(18.4 \%)$ & $85(24.1 \%)$ & \multirow[t]{7}{*}{$<0.0001^{4}$} & & \\
\hline . S-PD & $9(23.7 \%)$ & $23(6.5 \%)$ & & & \\
\hline . LST-GH & $2(5.3 \%)$ & $106(30.1 \%)$ & & & \\
\hline - LST-GM & $12(31.6 \%)$ & $89(25.3 \%)$ & & & \\
\hline - LST-NG-FE & $1(2.6 \%)$ & $36(10.2 \%)$ & & & \\
\hline - LST-NG-PD & $7(18.4 \%)$ & $13(3.7 \%)$ & & & \\
\hline - NA & 0 & 5 & & & \\
\hline Pseudodepression (+) & $25(65.8 \%)$ & $68(19 \%)$ & $<0.0001^{4}$ & OR 7.5 [3.6-15.6] & OR 4.6 [1.1-19.7] \\
\hline Adenomatous: Serrated & 38 (100\%): 0 (0\%) & 322 (90.2\%): 35 (9.8\%) & $0.0435^{4}$ & NS & NS \\
\hline \multicolumn{6}{|l|}{ JNET Classification ${ }^{5}$} \\
\hline - JNET 1 & $0(0 \%)$ & $21(7.8 \%)$ & \multirow[t]{5}{*}{$<0.0001^{4}$} & \multirow[t]{2}{*}{ I } & \multirow[t]{2}{*}{1} \\
\hline - JNET 2A & $8(30.8 \%)$ & $234(87.3 \%)$ & & & \\
\hline - JNET 2B & $15(57.7 \%)$ & $13(4.9 \%)$ & & \multirow[t]{2}{*}{ I } & \multirow[t]{2}{*}{ OR 51.8 [12.5-215.5] } \\
\hline - JNET 3 & $3(11.5 \%)$ & $0(0 \%)$ & & & \\
\hline . NA & 12 & 89 & & & \\
\hline
\end{tabular}

LST, laterally spreading tumor; S, sessile; PD, pseudodepression; GH, granular, homogeneous; GM, granular, mixed; NG-FE, non-granular-flat elevated; NG-PD, nongranular-pseudodepressed; NA, not available; NS, not significant; JNET, Japan NBI Expert Team Classification of vascular pattern.

${ }^{1}$ Model 1 (included observations $=390$ ): model fit $P<0.0001 ; \%$ of cases correctly classified $=90.26 \%$; AUC $=0.777$

${ }^{2}$ Model 2 (including JNET and non-lifting sign, included observations $=269$ ): model fit $P<0.0001 ; \%$ of cases correctly classified $=95.91 \%$; AUC $=0.914$

3 ROC curve analyses identified an age $\geq 74$ (Area under the curve $[A U C]=0.576, P=0.13$ ) and a diameter $\geq 40 \mathrm{~mm}(A \cup C=0.587, P=0.1$ ) as the cut-offs better separ-

ating an invasive versus a benign histology.

${ }^{4}$ statistically significant $(P<0.05)$

${ }^{5}$ proportion of lesions with available data 
- Table 2 Endoscopic management of lesions.

\begin{tabular}{|c|c|c|c|}
\hline Variable & $\begin{array}{l}\text { Invasive Lesions } \\
(\mathrm{N}=\mathbf{3 8})\end{array}$ & $\begin{array}{l}\text { Benign Lesions } \\
(\mathrm{N}=357)\end{array}$ & $P$ value \\
\hline Primary Surgery & $12(31.6 \%)$ & $34(9.5 \%)$ & $0.0002^{1}$ \\
\hline Endoscopic Treatment & $26(68.4 \%)$ & $323(90.5 \%)$ & $0.0002^{1}$ \\
\hline Non-lifting sign (+) & $6(23.1 \%)$ & $17(5.2 \%)$ & $0.0004^{1}$ \\
\hline \multicolumn{3}{|l|}{ Technique } & \multirow[t]{4}{*}{$0.0026^{1}$} \\
\hline - EMR & $16(61.5 \%)$ & $279(86.6 \%)$ & \\
\hline - ESD & $6(23.1 \%)$ & $24(7.5 \%)$ & \\
\hline - Hybrid techniques & $4(15.4 \%)$ & $19(5.9 \%)$ & \\
\hline \multicolumn{4}{|l|}{ Complications } \\
\hline - Perforations & $3(11.5 \%)$ & $6(1.9 \%)$ & $0.0028^{1}$ \\
\hline - Incomplete Resections & $10(38.5 \%)$ & $21(6.5 \%)$ & $<0.0001^{1}$ \\
\hline - EB : PM resection ${ }^{2}$ & $10(62.5 \%): 6(37.5 \%)$ & $148(49 \%): 154$ (51\%) & 0.2936 \\
\hline - Curative ER & $4(15.4 \%)$ & $302(93.5 \%)$ & $<0.0001^{1}$ \\
\hline - Non-curative ER & $22(84.6 \%)$ & $21(6.5 \%)$ & \\
\hline - $\mathrm{R}+$ & 9 & 21 & \\
\hline PM resections & 2 & & \\
\hline High-risk histology & 11 & & \\
\hline - $\mathrm{SMI}>1000 \mu \mathrm{m}$ & 7 & & \\
\hline - $\mathrm{LVI}+$ & 3 & & \\
\hline - G3 differentiation & 1 & & \\
\hline Complementary surgery ${ }^{3}$ & $18(69.2 \%)$ & $18(5.6 \%)$ & $<0.0001^{1}$ \\
\hline \multicolumn{4}{|c|}{ 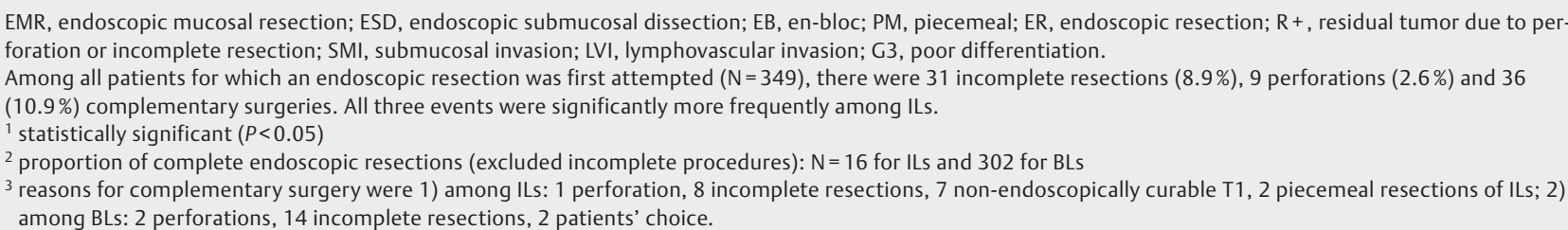 } \\
\hline
\end{tabular}

factors for recurrence were: PM resection $(31.2 \%$ vs $7.5 \%, P<$ $0.0001)$, a higher number of fragments $(9.6 \%, 23.5 \%$ and $30.9 \%$ for lesions removed EB, in 2 pieces and $\geq 3$ pieces, $P=$ 0.0014 ), involvement of "risky hotspots" and high-grade (HG) versus low-grade (LG) dysplasia (28.6\% vs $16.1 \%, P=0.0354$; - Table 3).

No association with resection techniques nor morphology was found.

\section{Multivariate logistic regression of variables associated with recurrence}

The involvement of "risky hotspots" (OR=6.3 [Cl 1.8-21.8]), a $\mathrm{PM}$ vs $\mathrm{EB}$ resection $(\mathrm{OR}=5.6[\mathrm{Cl} 2.3-13.7])$ and a $\mathrm{HG}$ vs $\mathrm{LG}$ dysplasia $(O R=2.7[\mathrm{Cl} 1.2-5.7])$ were found to be independent predictors of recurrence ( $\triangleright$ Table 3 and Supplementary Fig. 1).

\section{Cancer-specific mortality}

No CRC-related death was found in the whole cohort of patients with clinical follow-up (median = 36 months [IQR 19-70]).

Among patients lost to follow-up, three deaths were registered far from endoscopic resection (excluding endoscopyrelated events), with removed lesions further excluding cancer-specific mortality (20-25 mm LGD lesions, removed EB).

\section{Adjusted survival curves}

Adjusted rates of 1-year recurrence-free survival, surgery-free and CRC-free survival were $88.9 \%, 99.7 \%$, and $100 \%$, respectively. Five-year survival rates were $77.5 \%, 98.6 \%$, and $100 \%$, respectively. 
- Table 3 Comparisons of characteristics of recurrent versus not recurrent lesions during endoscopic follow-up.

\begin{tabular}{|c|c|c|c|c|c|}
\hline Variable & $\begin{array}{l}\text { Recurrent } \\
(N=42)\end{array}$ & $\begin{array}{l}\text { Not Recurrent } \\
(\mathrm{N}=163)\end{array}$ & $P$ value & $\begin{array}{l}\text { Prevalence of } \\
\text { recurrence } \\
\text { (\% of pts with } \\
\text { variable) }\end{array}$ & $\begin{array}{l}\text { Multivariate } \\
\text { analysis }{ }^{1} \\
\text { OR }[\mathrm{CI}]\end{array}$ \\
\hline Age [IQR], years & $69[61-74]$ & $69[61-74]$ & 0.8747 & & \\
\hline Dimensions [IQR], mm & $35[30-45]$ & $28[20-35]$ & $<0.0001^{2}$ & & NS \\
\hline Dimensions $\geq 30 \mathrm{~mm}^{3}$ & $33(78.6 \%)$ & $81(49.7 \%)$ & $0.0008^{2}$ & $28.9 \%$ vs. $9.9 \%$ & NS \\
\hline Colon : Rectum & $31(73.8 \%): 11(26.2 \%)$ & 114 (69.9\%): 49 (30.1\%) & 0.6238 & & \\
\hline Proximal : Distal & 24 (57.1\%): $18(42.9 \%)$ & 94 (57.7\%): $69(42.3 \%)$ & 0.9511 & & \\
\hline Sessile: LST & $9(21.4 \%): 33(78.6 \%)$ & 54 (33.1\%): 109 (66.9\%) & 0.1438 & & \\
\hline \multicolumn{3}{|l|}{ Composite Morphology } & \multirow[t]{7}{*}{0.1008} & & \\
\hline - S & $7(16.7 \%)$ & $40(24.5 \%)$ & & & \\
\hline - S-PD & $2(4.8 \%)$ & $14(8.6 \%)$ & & & \\
\hline - LST-GH & $18(42.9 \%)$ & $46(28.2 \%)$ & & & \\
\hline - LST-GM & $14(33.3 \%)$ & $39(23.9 \%)$ & & & \\
\hline - LST-NG-FE & $1(2.4 \%)$ & $18(11 \%)$ & & & \\
\hline - LST-NG-PD & $0(0 \%)$ & $6(3.7 \%)$ & & & \\
\hline Pseudodepression (+) & $9(21.4 \%)$ & $29(17.8 \%)$ & 0.5895 & & \\
\hline Adenomatous: Serrated & 41 (97.6\%): 1 (2.4\%) & 144 (88.3\%): $19(11.7 \%)$ & 0.0715 & $22.2 \%: 5 \%$ & NS \\
\hline \multicolumn{3}{|l|}{ Dysplasia } & \multirow[t]{2}{*}{0.0619} & & \\
\hline - LGD & $20(47.6 \%)$ & $104(63.8 \%)$ & & $16.1 \%$ & 1 \\
\hline . HGD & $22(52.4 \%)$ & $55(33.7 \%)$ & $\begin{array}{l}\text { HGD vs.LGD, } \\
p=0.0354^{2}\end{array}$ & $28.6 \%$ & OR $2.7[1.2-5.7]$ \\
\hline - $\mathrm{T} 1$ & $0(0 \%)$ & $4(2.5 \%)$ & & $0 \%$ & NS \\
\hline " PM: EB & 35 (83.3\%): 7 (16.7\%) & 77 (47.2\%): 86 (52.8) & $<0.0001^{2}$ & $31.2 \%: 7.5 \%$ & OR $5.6[2.3-13.7]$ \\
\hline \multicolumn{3}{|l|}{ Number of pieces } & \multirow[t]{4}{*}{$0.0014^{2}$} & & \multirow[t]{4}{*}{ NS } \\
\hline . 1 & $9(21.4 \%)$ & $85(52.1 \%)$ & & $9.6 \%$ & \\
\hline .2 & $4(9.5 \%)$ & $13(8 \%)$ & & $23.5 \%$ & \\
\hline . $\geq 3$ & $29(69 \%)$ & $65(39.9 \%)$ & & $30.9 \%$ & \\
\hline $\begin{array}{l}\text { Involvement of dentate } \\
\text { line. appendix. ileocecal } \\
\text { valve }(+)\end{array}$ & $7(16.7 \%)$ & $7(4.3 \%)$ & $0.0047^{2}$ & $50 \%$ vs. $18.3 \%$ & OR $6.3[1.8-21.8]$ \\
\hline \multicolumn{3}{|l|}{ Technique } & \multirow[t]{4}{*}{0.5066} & & \\
\hline - EMR & $36(85.7 \%)$ & $138(84.7 \%)$ & & & \\
\hline - ESD & $2(4.8 \%)$ & $15(9.2 \%)$ & & & \\
\hline - Hybrid procedures & $4(9.5 \%)$ & $10(6.1 \%)$ & & & \\
\hline \multicolumn{6}{|c|}{$\begin{array}{l}\text { LST, laterally spreading tumor; S, sessile; PD, pseudodepression; GH, granular, homogeneous; GM, granular, mixed; NG-FE, non-granular-flat elevated; NG- } \\
\text { granular-pseudodepressed; NA, not available; LGD, low-grade dysplasia; HGD, high-grade dysplasia; T1, submucosal invasion following TNM staging; EMR, } \\
\text { scopic mucosal resection; ESD, endoscopic submucosal dissection; EB, en-bloc resection; PM, piecemeal resection. } \\
\text { Involvement of risky hotspots, histology with HGD and a PM resection were independent significant predictors of recurrence at multivariate analysis. } \\
1 \text { model fit } P<0.0001 ; \% \text { of cases correctly classified }=80.98 \% \text {; AUC }=0.755 \\
{ }^{2} \text { statistically significant }(P<0.05) \\
\left.{ }^{3} \text { A ROC curve identified a dimension of the lesion } \geq 30 \mathrm{~mm} \text { as best (AUC }=0.702, P<0.0001\right) \text { separating recurrent }(28.9 \%) \text { vs. non-recurrent }(9.9 \%) \text { lesions } \\
(P=0.0008) \text {. }\end{array}$} \\
\hline
\end{tabular}



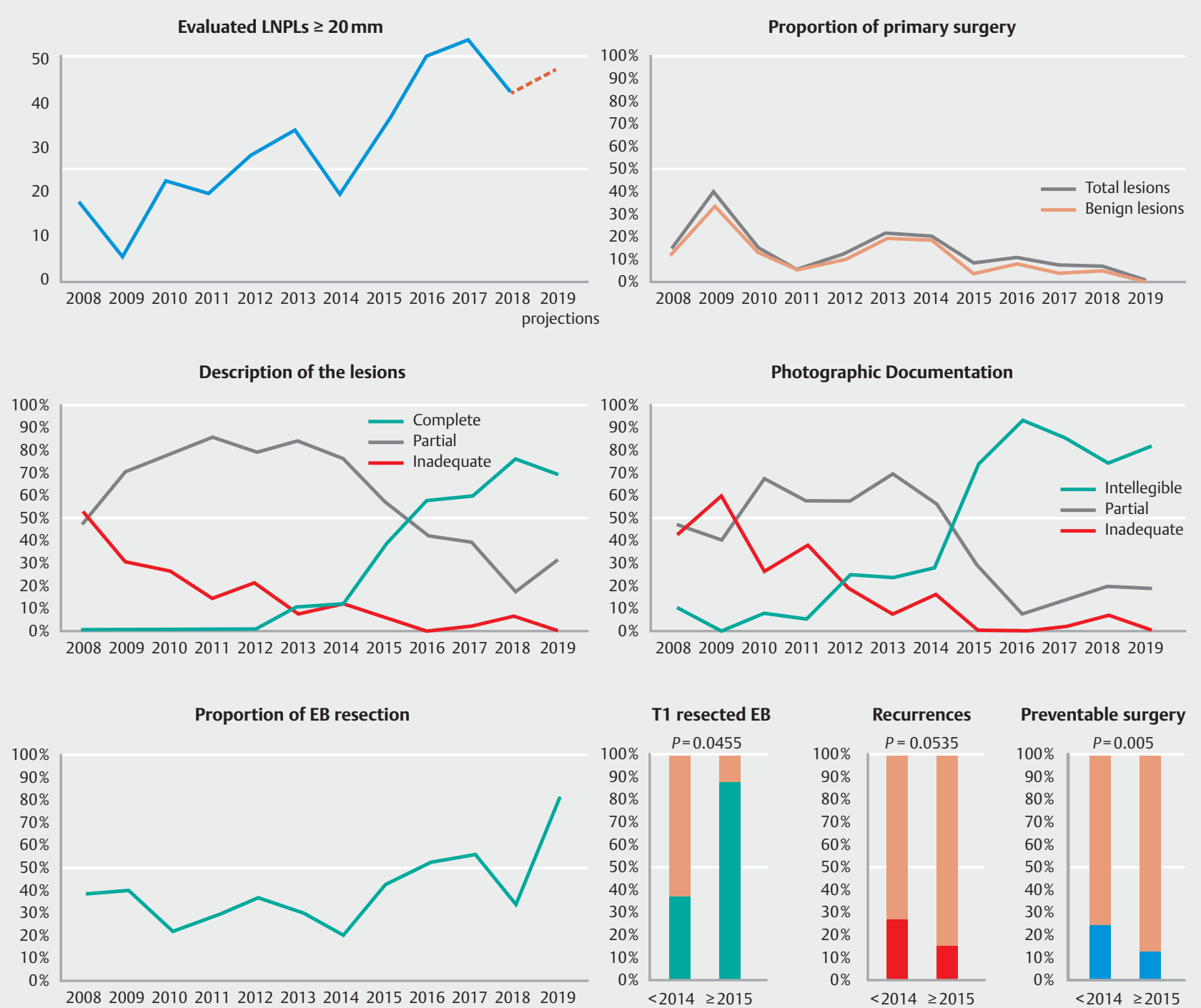

- Fig. 1 Temporal trends for performance measures. At the top-left, absolute number of evaluated large ( $\geq 20$ mm) non-pedunculated colorectal lesions. Subsequently: proportion of primary surgery, reporting of lesions, photographic documentation and proportion of en-bloc (EB) resection where represented as rate per year over the study period. At bottom-right, rates of submucosally invasive lesions resected EB, recurrent lesions and "potentially preventable surgery" separated according to two time intervals (before and after 2015, cut-off identified through ROC curve analysis as explained in the text).

\section{Surgically managed patients}

Among 88 operated patients, three $(3.4 \%)$ died within 30 days of surgery. All of them (median age $=71$ ) were in the group of primary elective surgery (SMI histology $3 / 3$ ).

\section{Trends over time}

Temporal trends of performance measures related to the assessment and management of LNPLs were evaluated graphically ( $\triangleright$ Fig. 1 ) and through ROC curve analysis ( $\triangleright$ Table 4$)$.

ROC curves repeatedly identified 2015 (following ESD introduction and education on pre-resective lesion assessment) as the year better separating procedures with a complete description of the lesion $(O R=37.8$ [16.9-84.4], $P<0.0001)$, an adequate iconographic documentation $(\mathrm{OR}=23.3[13.8-39.4], P<$
$0.0001)$ and availability of JNET classification ( $O R=13.43[7.3-$ 24.9 ], $P<0.0001)$. These 3 outcomes were present in $60.5 \%$, $82.3 \%$ and $93.5 \%$ of procedures $\geq 2015$.

After 2015, primary surgery was less indicated, both overall ( $7.5 \%$ vs $16.7 \%, P=0.0072$ ) and especially for BLs $(5.1 \%$ vs $14.8 \%, P=0.0019)$. EB removal of lesions with invasive histology was more frequent $(87.5 \%$ vs $37.5 \%, P=0.0455)$ and the rate of recurrence was lower $(15 \%$ vs $26 \% . P=0.0535)$. Finally, the overall rate of "potentially preventable" surgery was significantly lower $\geq 2015(12.6 \%$ vs $23.3 \%$. $P=0.005)$ ( Table 5$)$. 
- Table4 Temporal trends of performance measures.

\begin{tabular}{|c|c|c|c|c|}
\hline Outcome & Identified Cut-off & AUC & $P$ Value & Odds Ratios; $P$ value \\
\hline Adequate iconographic documentation & $\geq 2015$ & 0.840 & $<0.001^{1}$ & $\mathrm{OR}=23.3[13.8-39.4] .<0.0001$ \\
\hline $\begin{array}{l}\text { Completeness of report (location + morphology }+ \\
\text { dimensions }+ \text { superficial pattern) }\end{array}$ & $\geq 2015$ & 0.866 & $<0.0001^{1}$ & $\mathrm{OR}=37.8[16.9-84.4] .<0.0001$ \\
\hline JNET classification available & $\geq 2015$ & 0.801 & $<0.001^{1}$ & $\mathrm{OR}=13.43[7.3-24.9] .<0.0001$ \\
\hline Outpatients management of lesions & $\geq 2012$ & 0.564 & $<0.042^{1}$ & $\mathrm{OR}=3.1[1.5-6.1] \cdot 0.0015$ \\
\hline Primary surgery & $\geq 2015$ & 0.625 & $0.002^{1}$ & $\mathrm{OR}=0.4[0.2-0.8] \cdot 0.0055$ \\
\hline Benign lesions sent to primary surgery & $\geq 2015$ & 0.640 & $0.0022^{1}$ & $\mathrm{OR}=0.3[0.1-0.7] \cdot 0.0029$ \\
\hline Resections at index colonoscopy & $\geq 2019$ & 0.521 & NS & NS \\
\hline En-bloc removal of invasive lesions ${ }^{2}$ & $\geq 2015$ & 0.650 & NS & $\mathrm{OR}=11.7[0.9-147.6] \cdot 0.0578$ \\
\hline Incomplete resections & $\geq 2014$ & 0.587 & NS & NS \\
\hline Perforation & $\geq 2018$ & 0.510 & NS & NS \\
\hline Accurate histological stratification of $\mathrm{T} 1$ lesions & $\geq 2014$ & 0.600 & NS & NS \\
\hline Recurrence $^{3}$ & $\geq 2015$ & 0.571 & NS & $\mathrm{OR}=0.5[0.3-1] .0 .0554$ \\
\hline Surgery for recurrence ${ }^{3}$ & $\geq 2014$ & 0.598 & NS & NS \\
\hline "Non-curative endoscopic resection" & $\geq 2015$ & 0.563 & NS & $\mathrm{OR}=0.6[0.3-1.05] \cdot 0.0722$ \\
\hline "Potentially preventable surgeries" & $\geq 2015$ & 0.602 & $0.0043^{1}$ & $\mathrm{OR}=0.5[0.3-0.8] \cdot 0.0056$ \\
\hline \multicolumn{5}{|c|}{$\begin{array}{l}\text { JNET, Japan NBI Expert Team Classification of vascular pattern; NS, not significant. } \\
\text { We paired each procedure with a time lag (years) from inception of the study and analyzed this continuous variable through ROC curves to explore cut-offs separ- } \\
\text { ating periods with different prevalences of binary performance measures (ie, presence/absence of an outcome). For each outcome, the identified cut-off and sig- } \\
\text { nificance of the model are provided (as area under the curve (AUC and } P \text { value); In the rightmost column, odds ratios (OR) are reported, as a measure of the increased } \\
\text { or decreased probability to detect the outcome in the temporal interval following the identified cut-off. } \\
{ }^{1} \text { statistically significant ( } P<0.05 \text { ) } \\
2 \text { among patients with complete endoscopic resection } \\
3 \text { among patients with endoscopic resection undergoing follow-up }\end{array}$} \\
\hline
\end{tabular}

\section{Discussion}

According to our study, implementation of technological and educational interventions over time has resulted in a substantial improvement in endoscopic management of LNPLs in our center. The unique methodology used in this paper has underscored how not only specific interventions led to an increased confidence in handling these lesions and choosing the most appropriate therapeutic strategy, but even how this has translated into measurable changes in relevant clinical outcomes.

Endoscopic resection of these lesions often requires adoption of advanced endoscopic techniques (e. g. ESD), whose limits [4-6] impose a rigid selection of lesions [7, 27]. Characteristics associated with a higher probability of SMI have shown suboptimal accuracy, deriving from heterogeneous series, usually including specific subtypes of lesions (e.g. only LSTs $[1,28]$ ), specific resection techniques (e.g. only ESDs $[9,10]$ or EMRs $[12,19,29])$ and finally, not always including risk factors in a multivariate analysis $[9,12,30]$. In our series of consecutive LNPLs, potentially free of selection bias beyond referral, we confirmed the majority of known risk factors, with SMI rates according to single morphologies consistent with those summarized in a recent meta-analysis [1]. We further explored recently advocated factors (e.g. lesions distal to splenic flexure were morphologically more complex and histologically advanced [1, 30]), but when included in a multivariate model, only a few variables independently predicted SMI. Irregularity in superficial pit/vascular pattern seems to be the strongest SMI predictor. However, JNET classification was not available for all lesions, and published experiences have demonstrated that interobserver agreement is suboptimal, with specific training required to reach acceptable accuracy $[21,31,32]$. This could suggest that if the endoscopist has sufficient training/experience with pit/ vascular pattern interpretation, this should be the most considered parameter, along with presence of pseudodepression. However we also created a simpler model suggesting that size $\geq 40 \mathrm{~mm}$ and pseudodepression (evaluable without specific training) can nonetheless predict SMI histology better than other currently considered factors, including superficial morphology. Advance in the field of artificial intelligence will surely improve pre-resection assessment of LNPLs [19,33], integrating all morphological aspects into a risk estimate helping the endoscopist to adopt the most appropriate therapeutic strategy.

As for resection techniques, the dispute between ESD and EMR is still open. While larger/more specific series evaluating efficacy and safety of single techniques exist [12-14, 19,34- 
- Table 5 Differences in lesion characteristics, management, and outcomes before and after 2015.

\begin{tabular}{|c|c|c|c|}
\hline Variable & $\begin{array}{l}\text { Lesions managed }<2015 \\
(N=180)\end{array}$ & $\begin{array}{l}\text { Lesions managed } \geq 2015 \\
(\mathrm{~N}=215)\end{array}$ & $P$ value \\
\hline \multicolumn{4}{|l|}{ Assessment } \\
\hline Indication, screening & $32(17.8 \%)$ & $64(29.8 \%)$ & $0.0057^{1}$ \\
\hline Colon, rectum & 128 (71.7\%), 52 (28.9\%) & 167 (77.7\%), 48 (22.3\%) & 0.1357 \\
\hline Proximal, distal & 104 (57.8\%), 76 (42.2\%) & 136 (63.3\%), 79 (36.7\%) & 0.2674 \\
\hline Sessile, LST & $65(36.5 \%), 113(63.5 \%)$ & $59(27.4 \%), 156(72.6 \%)$ & 0.0543 \\
\hline \multicolumn{3}{|l|}{ Composite morphology } & \multirow[t]{8}{*}{$0.0169^{1}$} \\
\hline . $S$ & $47(26.7 \%)$ & $45(21 \%)$ & \\
\hline - S-PD & $18(10.2)$ & $14(6.5 \%)$ & \\
\hline - LST-GH & $47(26.7 \%)$ & $61(28.5 \%)$ & \\
\hline - LST-GM & $50(28.4 \%)$ & $51(23.8 \%)$ & \\
\hline - LST-NG-FE & $10(5.7 \%)$ & $27(12.6 \%)$ & \\
\hline - LST-NG-PD & $4(2.3 \%)$ & $16(7.5 \%)$ & \\
\hline - NA & 4 & 1 & \\
\hline \multicolumn{3}{|l|}{ Dysplasia } & \multirow[t]{4}{*}{$0.0384^{1}$} \\
\hline - LGD & $88(48.9 \%)$ & $133(61.9 \%)$ & \\
\hline - HGD/Tis & $74(41.1 \%)$ & $62(28.8 \%)$ & \\
\hline - T1 or superior & $18(10 \%)$ & $20(9.3 \%)$ & \\
\hline Pseudodepression (+) & $46(25.6 \%)$ & $47(21.9 \%)$ & 0.3893 \\
\hline Non-lifting sign (+) & $14(9.2 \%)$ & $9(4.5 \%)$ & 0.0820 \\
\hline Completeness of report & $7(3.9 \%)$ & $130(60.5 \%)$ & $<0.0001^{1}$ \\
\hline Adequate iconographic documentation & $30(16.7 \%)$ & $177(82.3 \%)$ & $<0.0001^{1}$ \\
\hline JNET classification available & $93(51.7 \%)$ & $201(93.5 \%)$ & $<0.0001^{1}$ \\
\hline Management regimen² & $N=149$ & $N=199$ & 0.1839 \\
\hline - Outpatients & $43(28.9 \%)$ & $75(37.7 \%)$ & \\
\hline - Day Hospital & $35(23.5 \%)$ & $46(23.1 \%)$ & \\
\hline - Hospitalized & $71(47.7 \%)$ & $78(39.2 \%)$ & \\
\hline \multicolumn{4}{|l|}{ Treatment } \\
\hline Primary surgery & $30(16.7 \%)$ & $16(7.5 \%)$ & $0.0072^{1}$ \\
\hline BLs sent to primary surgery & $24 / 162(14.8 \%)$ & $10 / 195(5.1 \%)$ & $0.0019^{1}$ \\
\hline Endoscopic treatment & $150(83.3 \%)$ & $199(92.6 \%)$ & $0.0072^{1}$ \\
\hline \multicolumn{3}{|l|}{ Endoscopic technique } & \multirow[t]{4}{*}{$<0.0001^{1}$} \\
\hline - EMR & $145(97.3 \%)$ & $150(75.4 \%)$ & \\
\hline - ESD & $1(0.7 \%)$ & $29(14.6 \%)$ & \\
\hline - Hybrid techniques & $3(2 \%)$ & $20(10.1 \%)$ & \\
\hline Perforation & $4(2.7 \%)$ & $5(2.5 \%)$ & 0.9285 \\
\hline Incomplete resections & $17(11.3 \%)$ & $14(7 \%)$ & 0.1629 \\
\hline EB resection ${ }^{3}$ & $54 / 133(40.6 \%)$ & $104 / 185(56.2 \%)$ & $0.0061^{1}$ \\
\hline EB removal of ILs ${ }^{3}$ & $3 / 8(37.5 \%)$ & $7 / 8(87.5 \%)$ & $0.0455^{1}$ \\
\hline Complementary surgery & $19(12.7 \%)$ & $17(8.5 \%)$ & 0.2105 \\
\hline
\end{tabular}


- Table 5 (Continuation)

\begin{tabular}{|c|c|c|c|}
\hline Variable & $\begin{array}{l}\text { Lesions managed }<2015 \\
(N=180)\end{array}$ & $\begin{array}{l}\text { Lesions managed } \geq 2015 \\
(\mathrm{~N}=215)\end{array}$ & $P$ value \\
\hline Recurrence $^{4}$ & $27 / 104(26 \%)$ & $15 / 100(15 \%)$ & 0.0535 \\
\hline Surgery for recurrence ${ }^{4}$ & $4 / 118(3.4 \%)$ & $2 / 127(1.6 \%)$ & 0.3594 \\
\hline “Non-curative” ER & $24 / 150(16 \%)$ & $19 / 199(9.5 \%)$ & 0.0698 \\
\hline "Potentially preventable surgery" & $42(23.3 \%)$ & $27(12.6 \%)$ & $0.0050^{1}$ \\
\hline \multicolumn{4}{|c|}{$\begin{array}{l}\text { LST, laterally spreading tumor; S, sessile; PD, pseudodepression; GH, granular, homogeneous; GM, granular, mixed; NG-FE, non-granular-flat elevated; NG-PD, non- } \\
\text { granular-pseudodepressed; NA, not available; LGD, low-grade dysplasia; HGD, high-grade dysplasia; T1, submucosal invasion following TNM staging; JNET, Japan NBI } \\
\text { Expert Team Classification of vascular pattern; EMR, endoscopic mucosal resection; ESD, endoscopic submucosal dissection; BLs, benign lesions; EB, en bloc; ILs, } \\
\text { invasive lesions; FU, follow-up; ER, endoscopic resection; PM, piecemeal. } \\
\text { From } 2015 \text { primary surgery was overall less indicated ( } 7.5 \% \text { vs. } 16.7 \%, P=0.0072) \text { as well as a lower proportion of benign lesions was sent to primary surgery ( } 5.1 \% \\
\text { vs. } 14.8 \%, P=0.0019) \text {. Moreover, the proportions of en-bloc removal of lesions with invasive histology was higher }(87.5 \% \text { vs. } 37.5 \%, P=0.0455) \text { and the rate of re- } \\
\text { currence lower ( } 15 \% \text { vs. } 26 \%, P=0.0535) \text {. The overall rate of "potentially preventable" surgeries was significantly lower }>2015(12.6 \% \text { vs. } 23.3 \% \text {. } P=0.005) \\
1 \text { statistically significant }(P<0.05) \\
2 \text { proportion of lesions with available data } \\
3 \text { proportion of complete endoscopic resection }\end{array}$} \\
\hline
\end{tabular}

39 , it remains difficult to demonstrate the impact of these techniques and of their learning curve on patients' long-term outcomes in real-life cohorts. In our experience, ESD was not per se associated with better outcomes but, compared to EMR, seems to have allowed the treatment of larger and more complex lesions. This was obviously paired with a higher need for subsequent surgery, due to a higher proportion of SMI lesions, a higher rate of AEs but above all to a higher detection of "highrisk" lesions for lymph node metastasis despite complete endoscopic resection. Of note, increasing the possibility of diagnosing NC-ERs at histology, to allow access to further treatments, is one precise expected advantage of ESD [40]. Taking all of this into consideration, at first glance, ESD seems to be a staging procedure, and the endoscopist must accept a non-irrelevant rate of subsequent surgery ( $17 \%$ in our series), which is closely connected to the greater complexity of lesions approached, for which the alternative would be a primary surgical referral. However, our data suggest that the rate of ILs for which surgery can be avoided thanks to ESD is not negligible, and is significantly higher than among EMRs. This is particularly important considering the lack of endoscopy-related and cancer-related mortality versus the $3.4 \%$ of surgery-related mortality in our cohort. In this scenario, every effort must be encouraged to attempt a primary ER for lesions without signs of deep SMI.

Endoscopic resection of LNPLs also involves the issue of recurrence, especially in the setting of PM resections $[12,16$, $41]$. In our cohort, we confirm that after complete ER, a recurrence may be found in almost one-quarter of patients, but is endoscopically manageable in the majority of cases. Even if our median endoscopic follow-up was relatively short (16 months [IQR 10-34]), data from the literature are reassuring because $96 \%$ of recurrences occur within 6 months after index resection, and only $2 \%$ of recurrences can be expected after 12 months [34], as also suggested by our median time-to-recurrence of 7 months (IQR 4-13). Our multivariate analysis identified independent predictors of recurrence, both known (PM vs
EB resection) and poorly reported (grading of dysplasia and involvement of "risky hotspots"). This may suggest that lesions with HG dysplasia or involving dentate line or ileocecal valve might deserve a tighter FU even when resected en bloc. The finding of recurrences after en bloc resections should not be a surprise, since "en-bloc" is an endoscopic definition, while histology must still be taken into account for subsequent patient management; moreover, histological definition of complete resection can be hampered by presence of cautery artefacts [42]; however, we cannot exclude a learning curve effect on these data.

We found that during the last 10 years, management of LNPLs in our center has significantly improved, with decreased surgical referral and more appropriate assessment and treatment of lesions with higher risk of SMI. We reached these conclusions after analyzing trends in performance measures over time through an original, but still precise, statistical method. This method identified the turning point of our quality improvement process to be in 2015, corresponding to about 1 year after the first rectal ESD was performed in our center, and probably linked to increasing confidence with the technique. Moreover, in 2015, an educational intervention on endoscopic pre-resection assessment was completed for both tutors and trainees, and our practice was audited and statistically assessed [40]. We strongly believe that these two aspects (technical and educational) go hand-in-hand in terms of improving proficiency of decision-making and management, as recommended by international position statements [4].

This study has limitations. First, even if the prospectively collected databases allowed the inclusion of all consecutive LNPLs, the retrospective nature did not permit evaluation of every outcome (e.g. minor complications). Second, as expected from a real-life series, included lesions were not homogeneous, potentially affecting analysis of risk factors and permitting only indirect considerations about resection techniques. Third, some 
parameters (JNET classification) were not available for all lesions.

Nevertheless, this study represents deep insight on real-life management of all consecutive LNPLs evaluated in a single institution, potentially free of any selection bias. All available records, images, and videos were blindly reevaluated to confirm morphology and superficial pattern. All lesions for which a pre-resection assessment is relevant to therapeutic strategy (i. e. both LSTs and sessile) were included, excluding only pedunculated polyps, which are always manageable EB. We used multivariate analyses in order to eliminate confounders. Moreover, while literature on quality improvement in this field is mainly about the learning curve of single resection techniques [8], this is the only available study in which statistical methods were adopted to evaluate temporal trends of performance measures relevant to patient outcomes.

\section{Conclusion}

In conclusion, management of LNPLs is still challenging and strongly depends on the ability to predict advanced histology and adverse outcomes, for which we provided multivariate models valuable both for primary screening centers and tertiary referral centers, further suggesting predictors to be explored in dedicated prospective cohorts. If SMI is no longer an a priori indication for surgery, it deserves advanced endoscopic management in referral centers, including advanced resection techniques. Reduction in primary surgical referrals is clearly paired with an increased need for secondary surgery both for complications and intrinsic histological risk, but nevertheless allows a net reduction in surgeries, and in their consequential burden in morbidity or mortality $[19,43]$. Centers that invest in education and technical improvements, with shared efforts by all involved specialists (endoscopists, surgeons, histopathologists) in a multidisciplinary setting, offer more appropriate and personalized management of LNPLs that leads to better outcomes for patients.

\section{Acknowledgements}

This study was supported by funds from Sapienza University Research Calls 2018.

\section{Competing interests}

The authors declare that they have no conflict of interest.

\section{References}

[1] Bogie R, Veldman M, Snijders L et al. Endoscopic subtypes of colorectal laterally spreading tumors (LSTs) and the risk of submucosal invasion: a meta-analysis. Endoscopy 2018; 50: 263-282

[2] Ferlitsch M, Moss A, Hassan C et al. Colorectal polypectomy and endoscopic mucosal resection (EMR): European Society of Gastrointestinal Endoscopy (ESGE) Clinical Guideline. Endoscopy 2017; 49: 270-297
[3] Rutter MD, Chattree A, Barbour JA et al. British Society of Gastroenterology/Association of Coloproctologists of Great Britain and Ireland guidelines for the management of large non-pedunculated colorectal polyps. Gut 2015; 64: 1847-1873

[4] Pimentel-Nunes P, Pioche M, Albéniz E et al. Curriculum for endoscopic submucosal dissection training in Europe: European Society of Gastrointestinal Endoscopy (ESGE) Position Statement. Endoscopy 2019; 51: 980-992

[5] Pimentel-Nunes P, Dinis-Ribeiro M, Ponchon T et al. Endoscopic submucosal dissection: European Society of Gastrointestinal Endoscopy (ESGE) Guideline. Endoscopy 2015; 47: 829-854

[6] Maple JT, Abu Dayyeh BK et al. ASGE Technology Committee JT. Endoscopic submucosal dissection. Gastrointest Endosc 2015; 81: $1311-1325$

[7] Draganov PV, Wang AY, Othman MO et al. AGA Institute Clinical Practice Update: Endoscopic Submucosal Dissection in the United States. Clin Gastroenterol Hepatol 2019; 17: 16-25.e1

[8] Grimm I, Peery AF, Kaltenbach T et al. Quality matters: improving the quality of care for patients with complex colorectal polyps. Am J Gastroenterol 2018; 113: 317-321

[9] Probst A, Ebigbo A, Märkl B et al. Endoscopic submucosal dissection for early rectal neoplasia: experience from a European center. Endoscopy 2016; 49: 222-232

[10] Yamada M, Saito Y, Sakamoto T et al. Endoscopic predictors of deep submucosal invasion in colorectal laterally spreading tumors. Endoscopy 2016; 48: 456-464

[11] Burgess NG, Hourigan LF, Zanati SA et al. Risk Stratification for covert invasive cancer among patients referred for colonic endoscopic mucosal resection: a large multicenter cohort. Gastroenterology 2017; 153: 732-742.e1

[12] Moss A, Bourke M], Williams SJ et al. Endoscopic mucosal resection outcomes and prediction of submucosal cancer from advanced colonic mucosal neoplasia. Gastroenterology 2011; 140: 1909-1918

[13] Repici A, Hassan C, De Paula Pessoa D et al. Efficacy and safety of endoscopic submucosal dissection for colorectal neoplasia: a systematic review. Endoscopy 2012; 44: 137-150

[14] Nakajima T, Saito Y, Tanaka S et al. Current status of endoscopic resection strategy for large, early colorectal neoplasia in Japan. Surg Endosc 2013; 27: 3262-3270

[15] Watanabe T, Muro K, Ajioka Y et al. Japanese Society for Cancer of the Colon and Rectum (JSCCR) guidelines 2016 for the treatment of colorectal cancer. Int J Clin Oncol 2018; 23: 1-34

[16] Oka S, Tanaka S, Saito Y et al. Local recurrence after endoscopic resection for large colorectal neoplasia: a multicenter prospective study in Japan. Am J Gastroenterol 2015; 110: 697-707

[17] Rönnow C-F, Uedo N, Toth E et al. Endoscopic submucosal dissection of 301 large colorectal neoplasias: outcome and learning curve from a specialized center in Europe. Endosc Int Open 2018; 06: E1340E1348

[18] Probst A, Golger D, Anthuber M et al. Endoscopic submucosal dissection in large sessile lesions of the rectosigmoid: Learning curve in a European center. Endoscopy 2012; 44: 660-667

[19] Milano RV, Viale E, Bartel M] et al. Resection outcomes and recurrence rates of endoscopic submucosal dissection (ESD) and hybrid ESD for colorectal tumors in a single Italian center. Surg Endosc 2018; 32: 2328-2339

[20] Bae JH, Yang DH, Lee S et al. Optimized hybrid endoscopic submucosal dissection for colorectal tumors: A randomized controlled trial. Gastrointest Endosc 2016; 83: 584-592

[21] Minoda $\mathrm{Y}$, Ogino $\mathrm{H}$, Chinen $\mathrm{T}$ et al. Objective validity of the Japan Narrow-Band Imaging Expert Team classification system for the differential diagnosis of colorectal polyps. Dig Endosc 2019; 31: 544551 
[22] Sano Y, Tanaka S, Kudo S et al. Narrow-band imaging (NBI) magnifying endoscopic classification of colorectal tumors proposed by the Japan NBI Expert Team. Dig Endosc 2016; 28: 526-533

[23] Endoscopic Classification Review Group. Update on the Paris Classification of superficial neoplastic lesions in the digestive tract. Endoscopy 2005; 37: 570-578

[24] Bosman FT. World Health Organization, International Agency for Research on Cancer. WHO classification of tumours of the digestive system. International Agency for Research on Cancer; 2010

[25] Beaton C, Twine CP, Williams GL et al. Systematic review and meta-analysis of histopathological factors influencing the risk of lymph node metastasis in early colorectal cancer. Color Dis 2013; 15: 788-797

[26] NCCN Clinical Practice Guidelines in Oncology. NCCN; 2019: Available at (accessed 28 September 2019): https://www.nccn.org/professionals/physician_gls/pdf/colon.pdf

[27] Bahin FF, Heitman SJ, Rasouli KN et al. Wide-field endoscopic mucosal resection versus endoscopic submucosal dissection for laterally spreading colorectal lesions: a cost-effectiveness analysis. Gut 2018; 67: 1965-1973

[28] Soliman H, Brieau B, Guillaumot M-A et al. Invasive pit pattern, macronodule and depression are predictive factors of submucosal invasion in colorectal laterally spreading tumours from a Western population. United Eur Gastroenterol J 2018; 6: 1569-1577

[29] Burgess NG, Hourigan LF, Zanati SA et al. Risk stratification for covert invasive cancer among patients referred for colonic endoscopic mucosal resection: a large multicenter cohort. Gastroenterology 2017; 153: 732-742.e1

[30] Miyamoto H, Ikematsu H, Fujii S et al. Clinicopathological differences of laterally spreading tumors arising in the colon and rectum. Int J Colorectal Dis 2014; 29: 1069-1075

[31] Sakamoto T, Takamaru H, Sekiguchi M et al. Reliability of Japan narrow-band imaging expert team classification for the diagnosis of colorectal neoplasms: a pilot study. Digestion 2019: 1-6

[32] Sumimoto K, Tanaka S, Shigita K et al. Diagnostic performance of Japan NBI Expert Team classification for differentiation among noninvasive, superficially invasive, and deeply invasive colorectal neoplasia. Gastrointest Endosc 2017; 86: 700-709
[33] Tamai N, Saito Y, Sakamoto T et al. Effectiveness of computer-aided diagnosis of colorectal lesions using novel software for magnifying narrow-band imaging: a pilot study. Endosc Int Open 2017; 05: E690E694

[34] Belderbos T, Leenders M, Moons L et al. Local recurrence after endoscopic mucosal resection of nonpedunculated colorectal lesions: systematic review and meta-analysis. Endoscopy 2014; 46: 388-402

[35] Repici A, Hassan C, Pagano $\mathrm{N}$ et al. High efficacy of endoscopic submucosal dissection for rectal laterally spreading tumors larger than $3 \mathrm{~cm}$. Gastrointest Endosc 2013; 77: 96-101

[36] Rönnow C-F, Uedo N, Toth E et al. Endoscopic submucosal dissection of 301 large colorectal neoplasias: outcome and learning curve from a specialized center in Europe. Endosc Int Open 2018; 06: E1340E1348

[37] Sauer M, Hildenbrand R, Oyama T et al. Endoscopic submucosal dissection for flat or sessile colorectal neoplasia $\geq 20 \mathrm{~mm}$ : A European single-center series of 182 cases. Endosc Int Open 2016; 04: E895E900

[38] Lee E-J, Lee JB, Lee SH et al. Endoscopic treatment of large colorectal tumors: comparison of endoscopic mucosal resection, endoscopic mucosal resection-precutting, and endoscopic submucosal dissection. Surg Endosc 2012; 26: 2220-2230

[39] Saito Y, Fukuzawa M, Matsuda T et al. Clinical outcome of endoscopic submucosal dissection versus endoscopic mucosal resection of large colorectal tumors as determined by curative resection. Surg Endosc 2010; 24: 343-352

[40] Margagnoni G, Angeletti S, D’Ambra G et al. Outcome and risk of recurrence for endoscopic resection of colonic superficial neoplastic lesions over $2 \mathrm{~cm}$ in diameter. Dig Liver Dis 2016; 48: 399-403

[41] Hwang JH, Konda V, Abu Dayyeh BK et al. Endoscopic mucosal resection. Gastrointest Endosc 2015; 82: 215-226

[42] Geramizadeh B, Owen DA. Handling and Pathology Reporting of Gastrointestinal Endoscopic Mucosal Resection. Middle East J Dig Dis 2017; 9: 5-11

[43] Kiriyama S, Saito Y, Yamamoto S et al. Comparison of endoscopic submucosal dissection with laparoscopic-assisted colorectal surgery for early-stage colorectal cancer: a retrospective analysis. Endoscopy 2012; 44: 1024-1030

\section{CORRECTION}

Giuseppe Vanella, Chiara Coluccio, Giulio Antonelli et al. Improving assessment and management of large non-pedunculated colorectal lesions in a Western center over 10 years: lessons learned and clinical impact Endoscopy International Open 2020; 08: E1252-E1263. DOI: $10.1055 / a-1220-6261$

In the paragraph Results on page E1254 interquartile range (IQR) was added two times.

In the paragraph recurrence on page E1254 interquartile range (IQR) was added two times.

In Table 1 to Multivariate $\mathrm{M}^{1}{ }^{1}$ and Multivariate $\mathrm{M} 2^{2} \mathrm{OR}$ (CI) was added. HR was changed into OR.

In Table 3 to Multivariate analysis $1 \mathrm{OR}(\mathrm{Cl})$ was added. HR was changed into OR.

In Fig. 1 values were corrected. Correct is: $P=0.0535$ and $P=0.005$; the a heading within the figure was changed in "Photographic Documentation".

In the footnotes of Table 5, in line 7 were changed the last figures in $12.6 \%$ vs $23.3 \%, P=0.005$. 